\title{
BMJ Open Fatty liver index, albuminuria and the association with chronic kidney disease: a population-based study in China
}

\author{
Kan Sun, ${ }^{1}$ Diaozhu Lin, ${ }^{1}$ Feng Li, ${ }^{1}$ Yiqin Qi, ${ }^{1}$ Wanting Feng, ${ }^{1}$ Li Yan, ${ }^{1}$ \\ Chaogang Chen, ${ }^{2}$ Meng Ren, ${ }^{1}$ Dan Liu ${ }^{1}$
}

To cite: Sun K, Lin D, Li F, et al. Fatty liver index, albuminuria and the association with chronic kidney disease: a populationbased study in China. BMJ Open 2018;8:e019097. doi:10.1136/

- Prepublication history for this paper is available online To view these files, please visit the journal online (http://dx.doi. org/10.1136/bmjopen-2017019097).

KS and DL contributed equally.

Received 11 August 2017 Revised 9 December 2017 Accepted 11 December 2017 bmjopen-2017-019097

\section{ABSTRACT}

Objectives The effects of lipid metabolism disorder on renal damage have drawn much attention. Using the fatty liver index (FLI) as a validated indicator of hepatic steatosis, this study aims to provide insight about the possible links between fatty liver and the development of chronic kidney disease (CKD).

Setting Hospital.

Participants We performed a population-based study on 9436 subjects aged 40 years or older.

Primary and secondary outcome measures FLI

is calculated using an algorithm based on body mass index, waist circumference, triglycerides and $\gamma$-glutamyltransferase. Increased urinary albumin excretion was defined according to the urinary albumin to creatinine ratio ranges $\geq 30 \mathrm{mg} / \mathrm{g}$. CKD was defined as estimated glomerular filtration rate (eGFR) $<60 \mathrm{~mL} / \mathrm{min} / 1.73 \mathrm{~m}^{2}$ or presence of albuminuria.

Results There were 620 (6.6\%) subjects categorised to have increased urinary albumin excretion and 753 (8.0\%) subjects categorised to have CKD. Participants with higher FLI had increased age, blood pressure, low-density lipoprotein cholesterol, fasting plasma glucose, fasting insulin and decreased eGFR level. Prevalence of increased urinary albumin excretion and CKD tended to increase with the elevated FLI quartiles. In logistic regression analysis, compared with subjects in the lowest quartile of FLI, the adjusted ORs in the highest quartile were $2.30(95 \% \mathrm{Cl}$ 1.36 to 3.90$)$ for increased urinary albumin excretion and 1.93 (95\% Cl 1.18 to 3.15 ) for CKD.

Conclusion Hepatic steatosis evaluated by FLI is independently associated with increased urinary albumin excretion and prevalence of CKD in middle-aged and elderly Chinese.

Check for updates

${ }^{1}$ Department of Endocrinology, Sun Yat-sen Memorial Hospital, Sun Yat-sen University,

Guangzhou, Republic of China ${ }^{2}$ Department of Nutrition, Sun Yat-sen Memorial Hospital, Sun Yat-sen University, Guangzhou, Republic of China

Correspondence to

Professor Dan Liu;

liudanye66@126.com
Chronic kidney disease (CKD) has become one of the leading public health problems worldwide. ${ }^{1} \mathrm{~A}$ recent national survey conducted between 2007 and 2010 reports that the prevalence of CKD was $10.8 \%$, representing an estimated 119.5 million patients in China with chronic kidney damage. ${ }^{2}$ In addition to CKD, an increasing number of studies have provided substantial evidence of albuminuria as a risk factor for future

\section{INTRODUCTION}

\section{Strengths and limitations of this study}

- The study was performed in a large populationbased cohort of 9436 Chinese subjects.

- Findings of the study may be applied to the majority of patients in general practice with suspected hepatic steatosis.

- Results should be interpreted cautiously due to the observational design of the current study.

cardiovascular events. ${ }^{3}$ Both renal and cardiovascular diseases sharing similar traditional risk factors, such as lipid metabolism disorder, could have particularly broad implications for the outcome of cardiovascular morbidity and mortality.

Association of hepatic steatosis with CKD development and its impact on the reduction of estimated glomerular filtration rate (eGFR) have been extensively investigated over the past decade. ${ }^{4}$ The substantial evidence linked hepatic steatosis to the increased risk and severity of CKD, which may be a target in the prevention and treatment of the disease. ${ }^{5}$ As a convenient scoring system for the presence of hepatic lipid deposits, the fatty liver index (FLI) is a surrogate steatosis biomarker developed in a cohort of patients from the general population. ${ }^{6}$ Compared with other techniques for evaluating hepatic steatosis, FLI is simple to obtain as body mass index (BMI), waist circumference (WC), triglycerides (TG) and $\gamma$-glutamyltransferase $(\gamma$-GGT) are routine measurements in clinical practice. Previous studies have demonstrated that FLI could determine fatty liver disease, incident type 2 diabetes and incident hypertension with considerable accuracy. ${ }^{6-8}$ Moreover, FLI is associated with insulin resistance, early atherosclerosis and risk of coronary heart disease, which could help physicians early detect subjects of greater cardiovascular risk and select patients for intensified lifestyle counselling. ${ }^{910}$ 
Clarifying the association of FLI with albuminuria and prevalent CKD would probably shed light on the prevention and pre-emptive treatment of related diseases. Recently, a cross-sectional study was conducted to investigate the association between FLI and CKD by recruiting adults undergoing a health check-up. ${ }^{11}$ However, by including only 731 subjects, the study did not evaluate the association between FLI and albuminuria, either. Therefore, we analysed data from a community-based Chinese population to comprehensively look into the relationship of FLI with both increased urinary albumin excretion and CKD.

\section{SUBJECTS AND METHODS}

\section{Study population and design}

We performed a cross-sectional study in a community in Guangzhou, China, from June to November 2011. The study population was from the Risk Evaluation of cAncers in Chinese diabeTic Individuals: a lONgitudinal (REACTION) study and the details of this study have been published previously. ${ }^{12-14}$ During the recruitment phase, a total of 10104 residents aged 40 years or older were invited to participate by examination notices or home visits. In total, 9916 subjects signed the consent form and agreed to participate in the survey. The participation rate was $98.1 \%$. Subjects who failed to provide information (BMI, n=206; WC, $\mathrm{n}=62$; TG, $\mathrm{n}=23$; $\gamma$-GGT, $\mathrm{n}=38$; or urinary albumin to creatinine ratio (ACR), $n=149$ ) were excluded from the analyses. Accordingly, a total of 9438 eligible individuals were included in the final data analyses. Written informed consent was obtained from each participant before data collection.

\section{Clinical and biochemical measurements}

We collected information on lifestyle factors, sociodemographic characteristics and family history using a standard questionnaire. Smoking or drinking habit was classified as 'never', 'current' (smoking or drinking regularly in the past 6 months) or 'ever' (cessation of smoking or drinking more than 6 months). ${ }^{15}$ A short form of the International Physical Activity Questionnaire was used to estimate physical activity at leisure time by adding questions on frequency and duration of moderate or vigorous activities and walking. ${ }^{16}$ Separate metabolic equivalent hours per week (MET-hour/week) were calculated to evaluate total physical activity.

All participants completed the anthropometrical measurements with the assistance of trained staff using standard protocols. Blood pressure measurements were obtained three times consecutively by the same observer at a 5 min interval using an automated electronic device (OMRON, Omron Company, Dalian, China). The average of three blood pressure measurements was used for analysis. Body height and body weight were recorded to the nearest $0.1 \mathrm{~cm}$ and $0.1 \mathrm{~kg}$ while participants were wearing light indoor clothing without shoes. BMI was calculated as weight in kilograms divided by height in metres squared $\left(\mathrm{kg} / \mathrm{m}^{2}\right)$. Obesity was defined as BMI $\geq 28$, and overweight was defined as BMI $\geq 24$ and $<28 .{ }^{17}$ WC was measured at the umbilical level with the participant in standing position, at the end of gentle expiration.

Venous blood samples were collected for laboratory tests after an overnight fasting of at least 10 hours. Measurement of fasting plasma glucose (FPG), fasting serum insulin, TG, total cholesterol (TC), high-density lipoprotein cholesterol (HDL-C), low-density lipoprotein cholesterol (LDL-C), creatinine, $\gamma$-GGT, aspartate aminotransferase (AST) and alanine aminotransferase (ALT) was done using an autoanalyser (Beckman CX-7 Biochemical Autoanalyzer, Brea, California, USA).

As surrogate marker of hepatic steatosis, FLI was analysed based on BMI, WC, TG and $\gamma$-GGT, and has been validated against liver ultrasound in the general population and proven accurate in detecting fatty liver. ${ }^{6}{ }^{10}$ FLI is calculated as the following: $\mathrm{FLI}=\left(\mathrm{e}^{0.953 \times \operatorname{loge}(\mathrm{TG})+0.139 \times}\right.$

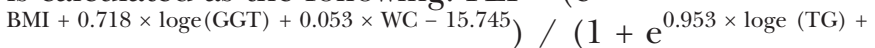
$0.139 \times \mathrm{BMI}+0.718 \times \operatorname{loge}(\mathrm{GGT})+0.053 \times \mathrm{WC}-15.745) \times 100$. The abbreviated Modification of Diet in Renal Disease formula recalibrated for Chinese population was used to calculate eGFR expressed in $\mathrm{mL} / \mathrm{min} / 1.73 \mathrm{~m}^{2}$ using the following formula: eGFR $=175 \times(\text { serum creatinine } \times 0.011)^{-1.234} \times$ $(\text { age })^{-0.179} \times(0.79$ if female $)$, where serum creatinine was expressed in $\mu \mathrm{mol} / \mathrm{L} .{ }^{18}$ Diabetes was diagnosed according to the 1999 WHO diagnostic criteria. ${ }^{19}$

\section{Definition of increased urinary albumin excretion, CKD and NAFLD}

Definitions of abnormalities in albumin excretion were according to the latest guidelines of the American Diabetes Association's Standards of Medical Care. ${ }^{20}$ The first morning spot urine samples were collected to assess the ACR. Urine albumin and creatinine were measured by chemiluminescence immunoassay (Siemens Immulite 2000, Orlando, Florida, USA) and the Jaffe's kinetic method (Biobase-Crystal, Jinan, China) on the automatic analyser, respectively. ACR was calculated by dividing the urinary albumin concentrations by the urinary creatinine concentrations, and is expressed in $\mathrm{mg} / \mathrm{g}$. The primary and secondary outcome measures were increased urinary albumin excretion and CKD, respectively. Increased urinary albumin excretion was defined according to the ACR ranges $\geq 30 \mathrm{mg} / \mathrm{g}$. CKD was defined as eGFR $<60 \mathrm{~mL} / \mathrm{min} / 1.73 \mathrm{~m}^{2}$ or presence of albuminuria (ACR $\geq 30 \mathrm{mg} / \mathrm{g}$ ). The optimal cut-off value of FLI in predicting non-alcoholic fatty liver disease (NAFLD) was 30 in Asian populations. ${ }^{21}$ Therefore, we classified the study population in non-current drinking group into NAFLD group (FLI $\geq 30$ ) and non-NAFLD group (FLI $<30)$.

\section{Statistical analysis}

Statistical analysis was performed using SAS V.9.2. Continuous variables were presented as mean $\pm \mathrm{SD}$ except for skewed variables, which were presented as median (IQR). Categorical variables were expressed as numbers (proportions). FLI, FPG, TG, ALT, AST, $\gamma$-GGT and MET-hour/ 
week were logarithmically transformed before analysis due to a non-normal distribution. FLI was presented as quartiles, and linear regression analysis was used to test for trend across groups. Differences among groups were tested by one-way analysis of variance, and post hoc comparisons were performed using Bonferroni correction. Comparisons between categorical variables were performed using the $\chi^{2}$ test.

Pearson's correlations were performed to test the correlations between FLI and the risk factors for kidney disease. Variables significant at $\mathrm{P}<0.20$ in Pearson's correlations were put into the multivariate stepwise linear regression models to identify factors that independently associated with FLI. We analysed the impact of FLI on the prevalence of increased urinary albumin excretion and CKD. Unadjusted and multivariate-adjusted logistic regression analyses were used to assess the risk of prevalent increased urinary albumin excretion and CKD in relation to each quartile increase in FLI level. Variables considered as potential covariates and significant in the stepwise linear regression were put into multivariateadjusted logistic regression analysis. Model 1 is unadjusted. Model 2 is adjusted for age. Model 3 is adjusted for age, sex, current smoking status, current drinking status, physical activity, systolic blood pressure (SBP), diastolic blood pressure (DBP), LDL-C, fasting insulin, ALT and AST. Model 4 is adjusted for age, sex, BMI, WC, current smoking status, current drinking status, physical activity, SBP, DBP, TG, LDL-C, fasting insulin, ALT, AST and $\gamma$-GGT. OR and the corresponding $95 \%$ CI were calculated. The relationship of FLI level with albuminuria and CKD was also explored in subgroups stratified by gender (men/women), age ( $\geq 60 /<60$ years), degree of obesity (normal/overweight/ obesity), current smoking (yes/no), current drinking (yes/ no), hypertension (yes/no) and diabetes (yes/no). Tests for interaction were performed by including simultaneously each strata factor, the quartiles of FLI level and the respective interaction terms (strata factor multiplied by quartiles of FLI level) in the models.

All statistical tests were two-sided, and a $\mathrm{P}$ value $<0.05$ was considered statistically significant.

\section{RESULTS}

\section{Clinical characteristics of the study population}

Among the 9436 enrolled individuals, the mean age was $55.9 \pm 8.0$ years. The median FLI was 19.1 with IQR of 8.6-37.4. There were $620(6.6 \%)$ subjects categorised to have increased urinary albumin excretion and 753 $(8.0 \%)$ subjects with $\mathrm{CKD}$, respectively. Table 1 shows the clinical and biochemical characteristics of the participants according to FLI quartiles. Participants with higher FLI level had increased age, BMI, WC, SBP, DBP, TG, TC, LDL-C, FPG, fasting insulin, ALT, AST and $\gamma$-GGT, and higher proportions of current smokers and current drinkers (all $\mathrm{P}$ for trend $<0.0001$ ). Higher FLI level also associated with decreased HDL-C and eGFR (all P for trend $<0.0001)$.

\section{Associations between $\mathrm{FLI}$ and metabolic risk factors}

Analysis of Pearson's correlation showed that age, sex, BMI, WC, SBP, DBP, TG, TC, HDL-C, LDL-C, FPG, fasting insulin, ALT, AST, $\gamma$-GGT and eGFR were significantly correlated with FLI level. Further multivariate stepwise linear regression showed that age, sex, BMI, WC, SBP, DBP, TG, LDL-C, fasting insulin, ALT, AST and $\gamma$-GGT were independent determinants of FLI level (table 2).

\section{Associations of FLI with increased urinary albumin excretion and CKD}

As shown in figure 1A, from the lowest quartile to the highest quartile of FLI level, the prevalence of increased urinary albumin excretion was $3.64 \%, 4.83 \%, 6.23 \%$ and $11.57 \%$, respectively ( $\mathrm{P}$ for trend $<0.0001$ ). Strikingly, the prevalence of CKD also tended to increase with the elevated FLI quartile (figure $1 \mathrm{~B}, \mathrm{P}$ for trend $<0.0001$ ). As shown in table 3 , compared with participants in quartile 1 of FLI, univariate logistic regression analysis showed that participants in quartile 2, quartile 3 and quartile 4, respectively, have a significant correlation with increased odds of increased urinary albumin excretion and CKD (all $\mathrm{P}$ for trend $<0.0001$ ). In multivariate logistic regression analyses (model 3), the ORs of increased urinary albumin excretion for increasing FLI quartiles were 1.00 (reference), 0.96 (95\% CI 0.66 to 1.39 ), 1.17 (95\% CI 0.77 to 1.77 ) and 2.30 (95\% CI 1.36 to 3.90 ). Similarly, the ORs of CKD for increasing FLI quartiles in model 3 were 1.00 (reference), 1.00 (95\% CI 0.71 to 1.40$), 1.03$ (95\% CI 0.70 to 1.51 ) and 1.93 (95\% CI 1.18 to 3.15 ), respectively (table 3 ). The prevalence of increased urinary albumin excretion was $51.6 \%$ and $29.6 \%$ in FLI-established NAFLD and non-NAFLD group $(\mathrm{P}<0.0001)$. Similar trends were detected in the prevalence of CKD (NAFLD group: 49.9\%; non-NAFLD group: $31.5 \%$; $\mathrm{P}<0.0001)$. Compared with the participants in the non-NAFLD group, those in the NAFLD group had higher prevalence of increased urinary albumin excretion (OR 1.58, 95\% CI 1.18 to 2.13 ) and CKD (OR $1.39,95 \%$ CI 1.05 to 1.82 ) in multivariate logistic regression analyses.

\section{Subgroup analysis of FLI with increased urinary albumin excretion and CKD}

As shown in figures 2 and 3, the associations of FLI level with increased urinary albumin excretion and CKD were not consistently the same in the subgroup analyses. A significant relationship of FLI level with both increased urinary albumin excretion and CKD was detected in women, younger subjects (age less than 60 years), overweight subjects, non-current smokers, non-current drinkers, and in those with hypertension or diabetes (all $\mathrm{P}<0.05$ ). In the subgroup analysis, no statistically significant interaction term between quartiles of FLI and each strata factor was detected.

\section{DISCUSSION}

We evaluated the association between hepatic steatosis and kidney disease in a large population of middle-aged 


\begin{tabular}{|c|c|c|c|c|c|}
\hline & Quartile 1 & Quartile 2 & Quartile 3 & Quartile 4 & P for trend \\
\hline n (\%) & $2360(25.01)$ & 2359 (24.99) & 2359 (24.99) & $2360(25.01)$ & \\
\hline Fatty liver index & $5.00(3.27-6.74)$ & $13.23(10.76-15.99)$ & $26.96(22.74-31.75)$ & $54.71(45.40-68.10)$ & \\
\hline $\begin{array}{l}\text { Urinary albumin to } \\
\text { creatinine ratio }(\mathrm{mg} / \mathrm{g})\end{array}$ & 7.65 (5.59-11.12) & $8.01(5.64-11.71)$ & $8.06(5.73-11.83)^{\star}$ & $8.93(5.96-15.01)^{\star} † \ddagger$ & $<0.0001$ \\
\hline Age (years) & $54.3 \pm 7.8$ & $55.8 \pm 7.9^{*}$ & $56.5 \pm 7.9^{*} \dagger$ & $56.9 \pm 8.3^{*} \dagger$ & $<0.0001$ \\
\hline Male, n (\%) & 427 (18.09) & $593(25.17)$ & $701(29.72)$ & 975 (41.31) & $<0.0001$ \\
\hline $\mathrm{BMI}\left(\mathrm{kg} / \mathrm{m}^{2}\right)$ & $20.6 \pm 2.0$ & $22.9 \pm 2.0^{*} \ddagger$ & $24.4 \pm 2.1^{*} \dagger$ & $26.8 \pm 3.5^{\star} † \ddagger$ & $<0.0001$ \\
\hline WC (cm) & $72.0 \pm 5.8$ & $79.3 \pm 5.4^{*} \ddagger$ & $84.1 \pm 5.5^{\star} \dagger$ & $91.3 \pm 8.5^{\star} \dagger \ddagger$ & $<0.0001$ \\
\hline $\mathrm{SBP}(\mathrm{mm} \mathrm{Hg})$ & $118.6 \pm 14.7$ & $124.5 \pm 15.9^{*} \ddagger$ & $128.4 \pm 15.8^{\star} \dagger$ & $132.5 \pm 16.1^{*} † \ddagger$ & $<0.0001$ \\
\hline $\mathrm{DBP}(\mathrm{mm} \mathrm{Hg})$ & $71.2 \pm 9.1$ & $74.2 \pm 9.3^{*} \ddagger$ & $76.5 \pm 9.4^{*} \dagger$ & $79.3 \pm 9.8^{*} † \ddagger$ & $<0.0001$ \\
\hline Current smoking, n (\%) & $169(7.3)$ & $202(8.7)$ & $227(9.8)$ & $335(14.4)$ & $<0.0001$ \\
\hline Current drinking, n (\%) & $57(2.5)$ & $70(3.0)$ & $68(2.9)$ & $117(5.1)$ & $<0.0001$ \\
\hline $\mathrm{TG}(\mathrm{mmol} / \mathrm{L})$ & $0.85(0.69-1.07)$ & $1.12(0.90-1.43)^{\star} \ddagger$ & $1.49(1.13-1.94)^{\star} \dagger$ & $2.10(1.56-3.01)^{\star} \dagger \ddagger$ & $<0.0001$ \\
\hline $\mathrm{TC}(\mathrm{mmol} / \mathrm{L})$ & $4.79 \pm 1.24$ & $5.16 \pm 1.22^{*} \ddagger$ & $5.35 \pm 1.13^{*} \dagger$ & $5.54 \pm 1.17^{*} † \ddagger$ & $<0.0001$ \\
\hline $\mathrm{HDL}-\mathrm{C}(\mathrm{mmol} / \mathrm{L})$ & $1.45 \pm 0.41$ & $1.37 \pm 0.35^{\star} \ddagger$ & $1.29 \pm 0.31^{*} \dagger$ & $1.19 \pm 0.28^{\star} \dagger \ddagger$ & $<0.0001$ \\
\hline LDL-C (mmol/L) & $2.82 \pm 0.90$ & $3.19 \pm 0.94^{\star} \ddagger$ & $3.31 \pm 0.91^{*} \dagger$ & $3.28 \pm 0.95^{\star} \dagger$ & $<0.0001$ \\
\hline FPG (mmol/L) & $5.23(4.89-5.61)$ & $5.33(4.95-5.80)^{\star} \ddagger$ & $5.47(5.05-5.96)^{*} \dagger$ & $5.73(5.23-6.42)^{\star} \dagger \ddagger$ & $<0.0001$ \\
\hline Fasting insulin ( $\mu \mathrm{lU} / \mathrm{mL})$ & $5.10(3.90-6.50)$ & $6.50(5.00-8.40)^{\star} \mp$ & $7.90(6.10-10.30)^{\star} \dagger$ & $10.50(7.80-13.70)^{\star} \dagger \ddagger$ & $<0.0001$ \\
\hline ALT (U/L) & $10.0(8.0-14.0)$ & $12.0(9.0-16.0)^{\star} \ddagger$ & $13.0(10.0-17.0)^{\star} \dagger$ & $17.0(12.0-24.0)^{\star} † \ddagger$ & $<0.0001$ \\
\hline AST (U/L) & $17.0(14.0-20.0)$ & $18.0(15.0-21.0)^{\star} \ddagger$ & $18.0(15.0-22.0)^{*} \dagger$ & $20.0(17.0-25.0)^{\star} \dagger \ddagger$ & $<0.0001$ \\
\hline$\gamma$-GGT (U/L) & $14.0(11.0-17.0)$ & $18.0(14.0-23.0)^{\star} \ddagger$ & $22.0(17.0-29.0)^{\star} \dagger$ & $31.0(23.0-47.0)^{\star} \dagger \ddagger$ & $<0.0001$ \\
\hline $\begin{array}{l}\text { Serum creatinine } \\
(\mu \mathrm{mol} / \mathrm{L})\end{array}$ & $65.3 \pm 15.5$ & $68.8 \pm 16.0^{*} \ddagger$ & $70.5 \pm 16.0^{*} \dagger$ & $74.9 \pm 17.2^{*} † \neq$ & $<0.0001$ \\
\hline eGFR (mL/min/1.73 m²) & $108.0 \pm 25.4$ & $102.5 \pm 23.7^{\star} \ddagger$ & $99.9 \pm 19.6^{*} \dagger$ & $95.5 \pm 19.5^{\star} \dagger \ddagger$ & $<0.0001$ \\
\hline $\begin{array}{l}\text { Physical activity (MET- } \\
\text { hour/week) }\end{array}$ & $24.0(10.5-49.0)$ & $24.0(10.5-45.0)$ & $23.0(10.5-42.0)$ & $21.0(10.5-42.0)^{\star}$ & 0.006 \\
\hline
\end{tabular}

Data were mean $\pm S D$ or median $(\mathrm{IQR})$ for skewed variables or numbers (proportions) for categorical variables.

$\mathrm{P}$ for trend was calculated for the linear regression analysis tests across the groups. $\mathrm{P}$ values were for the analysis of variance or $\chi^{2}$ analyses across the groups.

${ }^{*} \mathrm{P}<0.05$ compared with quartile 1 of fatty liver index.

$\dagger P<0.05$ compared with quartile 2 of fatty liver index.

$\ddagger \mathrm{P}<0.05$ compared with quartile 3 of fatty liver index.

ALT, alanine aminotransferase; AST, aspartate aminotransferase; BMI, body mass index; DBP, diastolic blood pressure; eGFR, estimated glomerular filtration rate; FLI, fatty liver index; FPG, fasting plasma glucose; HDL-C, high-density lipoprotein cholesterol; LDL-C, low-density lipoprotein cholesterol; MET-hour/week, metabolic equivalent hours per week; SBP, systolic blood pressure; TC, total cholesterol; TG, triglycerides; WC, waist circumference; $\gamma$-GGT, $\gamma$-glutamyltransferase.

Chinese subjects from the REACTION study. The presence of fatty liver assessed by FLI was associated with increased urinary albumin excretion and reduction of eGFR in the present study. The association was independent of potential confounding risk factors. To our current knowledge, this is the largest population-based study to explore the association of FLI with both albuminuria and CKD in Asian population. Early intervention is of great importance for albuminuria and CKD, and the present findings may just give insights into lipid metabolism for the prevention and early detection of the diseases.

The problem of obesity and NAFLD is now increasingly recognised in the Asian population. Prevalence of obesity was $7.9 \%$ ( $8.4 \%$ in men and $7.6 \%$ in women) in southern China, which has increased dramatically over the past several decades. ${ }^{22}$ There is a strong correlation between established obesity and incidence of NAFLD. Pooled prevalence of NAFLD diagnosed by ultrasound, CT scan and MRI was estimated to be $27.4 \%$ in subjects from Asian countries aged over 30 years. ${ }^{23}$ Even among the non-obese Chinese, $8.9 \%$ developed NAFLD in 5 years from 2006 to $2011 .{ }^{24}$ Therefore, early and accurate diagnosis of NAFLD is of great importance. The best method for an accurate assessment and diagnosis of hepatic steatosis is histological analysis of biopsies. ${ }^{25}$ However, it is uneconomical to conduct liver biopsies especially because of our large sample population. Hepatic ultrasonic examination is widely used in clinical practice and 
Table 2 Pearson's correlation and stepwise regression analysis of determinants of FLI

\begin{tabular}{|c|c|c|c|c|}
\hline & $\mathbf{r}$ & $P$ value & Standardised $\beta$ & $P$ value \\
\hline Age (years) & 0.12 & $<0.0001$ & 0.01 & 0.010 \\
\hline Sex (men=1, women=2) & -0.19 & $<0.0001$ & -0.04 & $<0.0001$ \\
\hline WC (cm) & 0.78 & $<0.0001$ & 0.42 & $<0.0001$ \\
\hline Physical activity (MET-hour/week) & -0.02 & 0.060 & - & - \\
\hline $\mathrm{DBP}(\mathrm{mm} \mathrm{Hg})$ & 0.32 & $<0.0001$ & 0.01 & 0.047 \\
\hline $\mathrm{TG}(\mathrm{mmol} / \mathrm{L})$ & 0.68 & $<0.0001$ & 0.42 & $<0.0001$ \\
\hline HDL-C (mmol/L) & -0.26 & $<0.0001$ & - & - \\
\hline LDL-C (mmol/L) & 0.21 & $<0.0001$ & 0.06 & $<0.0001$ \\
\hline FPG (mmol/L) & 0.22 & $<0.0001$ & - & - \\
\hline AST (U/L) & 0.15 & $<0.0001$ & -0.03 & $<0.0001$ \\
\hline$\gamma$-GGT (U/L) & 0.35 & $<0.0001$ & 0.16 & $<0.0001$ \\
\hline eGFR (mL/min/1.73 m²) & -0.19 & $<0.0001$ & - & - \\
\hline
\end{tabular}

ALT, alanine aminotransferase; AST, aspartate aminotransferase; $\beta$, regression coefficient; BMI, body mass index; DBP, diastolic blood pressure; eGFR, estimated glomerular filtration rate; FLI, fatty liver index; FPG, fasting plasma glucose; HDL-C, high-density lipoprotein cholesterol; LDL-C, low-density lipoprotein cholesterol; MET-hour/week, metabolic equivalent hours per week; r, correlation coefficient; SBP, systolic blood pressure; TG, triglycerides; WC, waist circumference; $\gamma$-GGT, $\gamma$-glutamyltransferase.

epidemiological studies in detecting fatty infiltration of the liver. ${ }^{26}{ }^{27}$ However, the non-invasive technique is not sensitive enough to detect mild steatosis and does not allow precise quantification of severity of steatosis in hepatic tissue. ${ }^{28}$

As another surrogate marker of histological fatty liver, FLI is defined as the accumulation of excessive liver fat. ${ }^{29}$ Based on previous research, FLI has been proven accurate in detecting fatty liver against liver ultrasound and in demonstrating the presence of hepatic fat against magnetic resonance spectroscopy. ${ }^{691021}$ The superiority of this non-invasive assessment techniques is that a higher score will indicate a higher degree of steatosis in hepatic tissue. However, an optimal cut-off point of the FLI in evaluating liver fatty infiltration should be considered as it varied according to the study population. ${ }^{21} 30$ Originally, FLI $>60$ was suggested to rule in NAFLD in Caucasian subjects. However, the optimal cut-off value of FLI in predicting NAFLD was different in Asian populations. In one recent study, Huang $e t a l^{21}$ found that FLI could accurately identify NAFLD and the optimal cut-off point was 30 in middle-aged and elderly Chinese. FLI could also accurately identify ultrasonography fatty liver in a large-scale population in Taiwan but with different optimal cut-off values, while an FLI $>35$ for men and $>20$ for women rule in NAFLD in their study. ${ }^{30}$ Based on the results of our research on Chinese subjects, further studies are needed to externally discuss the optimal cut-off point of the FLI in predicting hepatic steatosis.

Detection and prevention of kidney disease progression and urinary albumin excretion are difficult to process in the early stage. Dyslipidaemia is increasingly recognised as an important pathogenic mechanism in the deterioration of renal function. Recently, we conducted a clinical investigation to assess the associations of routine lipid measures with kidney disease in the same cohort. In the study, discordant associations of lipid parameters with renal insufficiency were detected, while the TG to HDL-C ratio is a better marker for evaluating increased urinary albumin excretion and CKD. ${ }^{31}$ As one of the phenotypes of dyslipidaemia, the pathogenesis of hepatic steatosis is closely related to kidney disease with regard to insulin resistance and chronic inflammation..$^{32}$ Based on recent studies, hepatokines, which are proteins secreted by hepatocytes, have been found to be linked to the induction of metabolic phenotypes through interorgan communication. ${ }^{33}$ Because of the high prevalence and burden of the fatty liver disease, it is important to identify which patients are most likely to be exposed to early-stage renal injury. ${ }^{23}$ Consequently, we closely monitor the association of hepatic steatosis predicted by FLI with prevalent increased urinary albumin excretion and CKD.

Consistent with our findings, a previous study reported that hepatic steatosis evaluated by FLI might contribute to CKD development. ${ }^{11}$ Elevated albuminuria is well known to be associated with increased risk for early diabetes renal damage; however, the identification and classification of kidney disease were assessed only by eGFR in that study. Moreover, 731 adults who underwent routine health evaluations were included in that study and the small sample size cannot better represent the whole population. By totally including 9438 subjects 
(A)

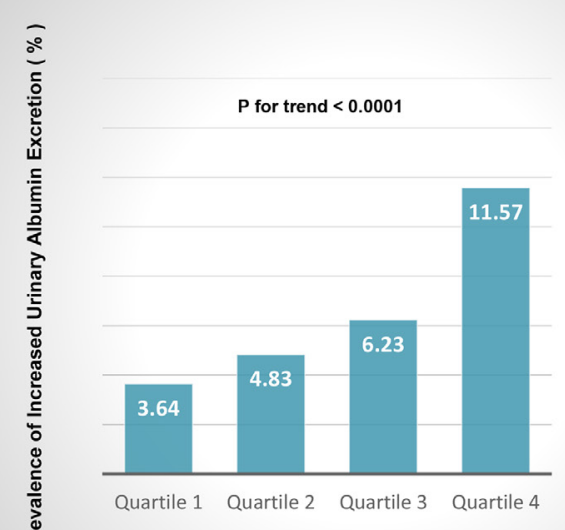

Quartiles of Fatty Liver Index

(B)

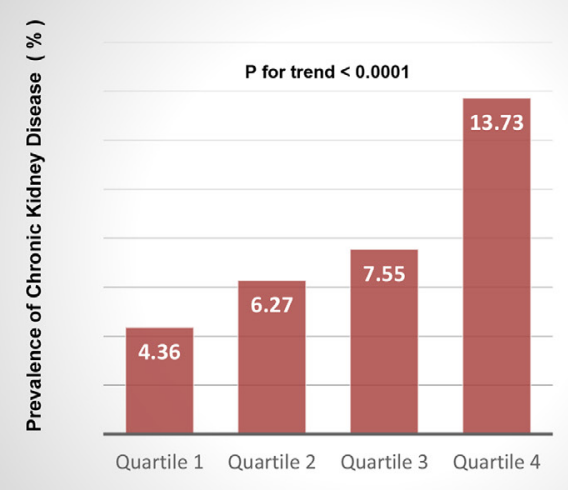

Quartiles of Fatty Liver Index

Figure 1 Prevalence of increased urinary albumin excretion and chronic kidney disease (CKD) in different quartiles of fatty liver index levels: (A) increased urinary albumin excretion and (B) CKD.

and adopting both albuminuria and eGFR for renal damage assessment, data in our study demonstrated that the FLI is associated with kidney disease, which might be an efficient screening indicator for the early prevention of related diseases in Chinese subjects. Recently, an interesting study by Giorda $e t a l^{34}$ reported that NAFLD is a dynamic condition in subjects with type 2 diabetes, and about 5\% Italian patients with diabetes entering or leaving FLI assessed NAFLD status every year. They found that male sex and established organ damage, especially kidney function, were independent risk predictors for the dynamic NAFLD condition in a longitudinal 3-year analysis. Because of the similarity in traditional risk factors for both NAFLD and CKD, the relationship between the prevalence of earlier stages of kidney damage and the incidence of NAFLD is complex. Longitudinal observation of our cohort needs to be carried out to determine whether such dynamic condition existed in Chinese, especially in those with type 2 diabetes.

Alcohol consumption can profoundly disturb lipid metabolism, which has prominent effects on hepatic tissue steatosis and insulin sensitivity. ${ }^{35}$ However, potential health effects regarding alcohol consumption in this field is also worth attaching attention. A meta-analysis of intervention studies by Schrieks et $a \hat{l}^{36}$ showed that moderate alcohol intake could improve insulin sensitivity by decreasing fasting insulin level in women. Recently, a prospective cohort study found that alcohol consumption was consistently inversely associated with urinary albumin excretion and the risk of developing CKD. ${ }^{37}$ Therefore, advice on alcohol consumption to subjects with low-grade hepatic tissue steatosis should consider the full range of benefits and risks, especially among those who drink moderately.

Table 3 Risk of prevalent albuminuria and CKD according to quartiles of FLI

\begin{tabular}{|c|c|c|c|c|c|c|}
\hline & & Quartile 1 & Quartile 2 & Quartile 3 & Quartile 4 & P for trend \\
\hline \multirow{4}{*}{$\begin{array}{l}\text { Increased urinary } \\
\text { albumin excretion }\end{array}$} & Model 1 & 1 & 1.34 (1.01 to 1.79$)$ & 1.76 (1.34 to 2.31$)$ & 3.46 (2.70 to 4.44$)$ & $<0.0001$ \\
\hline & Model 2 & 1 & 1.29 (0.97 to 1.72$)$ & 1.66 (1.27 to 2.19$)$ & 3.25 (2.53 to 4.17$)$ & $<0.0001$ \\
\hline & Model 3 & 1 & 0.94 (0.66 to 1.33$)$ & $1.13(0.81$ to 1.59$)$ & 2.22 (1.60 to 3.07$)$ & $<0.0001$ \\
\hline & Model 4 & 1 & 0.96 (0.66 to 1.39$)$ & $1.17(0.77$ to 1.77$)$ & $2.30(1.36$ to 3.90$)$ & 0.001 \\
\hline \multirow[t]{4}{*}{ CKD } & Model 1 & 1 & $1.47(1.13$ to 1.90$)$ & 1.79 (1.39 to 2.30$)$ & 3.49 (2.77 to 4.39$)$ & $<0.0001$ \\
\hline & Model 2 & 1 & 1.39 (1.07 to 1.80$)$ & 1.65 (1.28 to 2.12$)$ & 3.16 (2.51 to 3.99$)$ & $<0.0001$ \\
\hline & Model 3 & 1 & 0.99 (0.73 to 1.36$)$ & 1.03 (0.75 to 1.40$)$ & 1.95 (1.44 to 2.64$)$ & $<0.0001$ \\
\hline & Model 4 & 1 & 1.00 (0.71 to 1.40$)$ & $1.03(0.70$ to 1.51$)$ & $1.93(1.18$ to 3.15$)$ & 0.012 \\
\hline
\end{tabular}

Data are ORs $(95 \% \mathrm{Cl})$. Participants without increased urinary albumin excretion or CKD are defined as 0 and with increased urinary albumin excretion or CKD as 1.

Model 1 is unadjusted.

Model 2 is adjusted for age.

Model 3 is adjusted for age, sex, current smoking status, current drinking status, physical activity, SBP, DBP, LDL-C, fasting insulin, ALT and AST.

Model 4 is adjusted for age, sex, BMI, WC, current smoking status, current drinking status, physical activity, SBP, DBP, TG, LDL-C, fasting insulin, ALT, AST and $\gamma$-GGT.

ALT, alanine aminotransferase; AST, aspartate aminotransferase; BMI, body mass index; CKD, chronic kidney disease; DBP, diastolic blood pressure; FLI, fatty liver index; FPG, fasting plasma glucose; LDL-C, low-density lipoprotein cholesterol; SBP, systolic blood pressure; TG, triglycerides; WC, waist circumference; $\gamma$-GGT, $\gamma$-glutamyltransferase. 


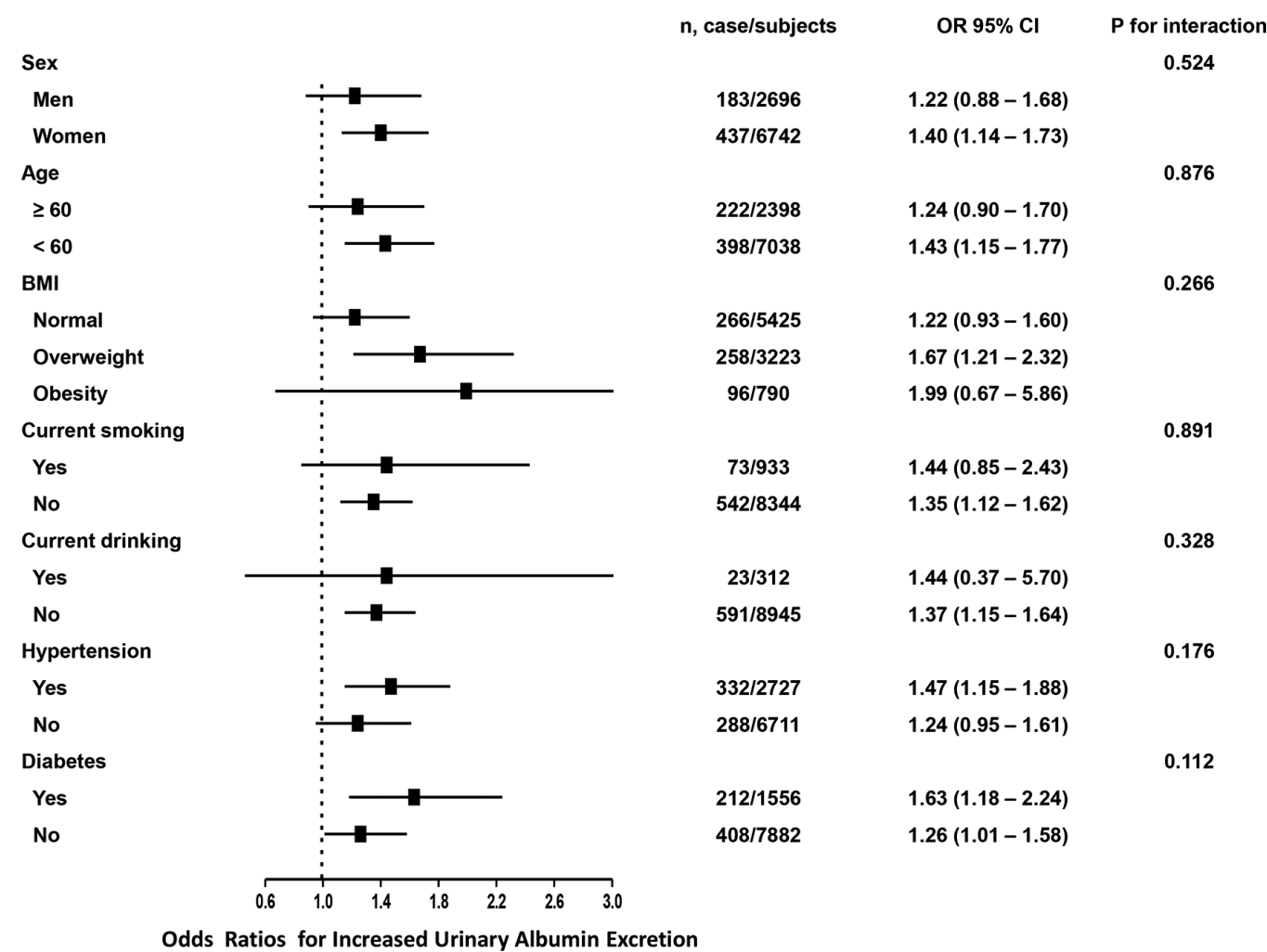

Figure 2 Risk of prevalent increased urinary albumin excretion with each quartile increase of fatty liver index levels in different subgroups. BMI, body mass index.

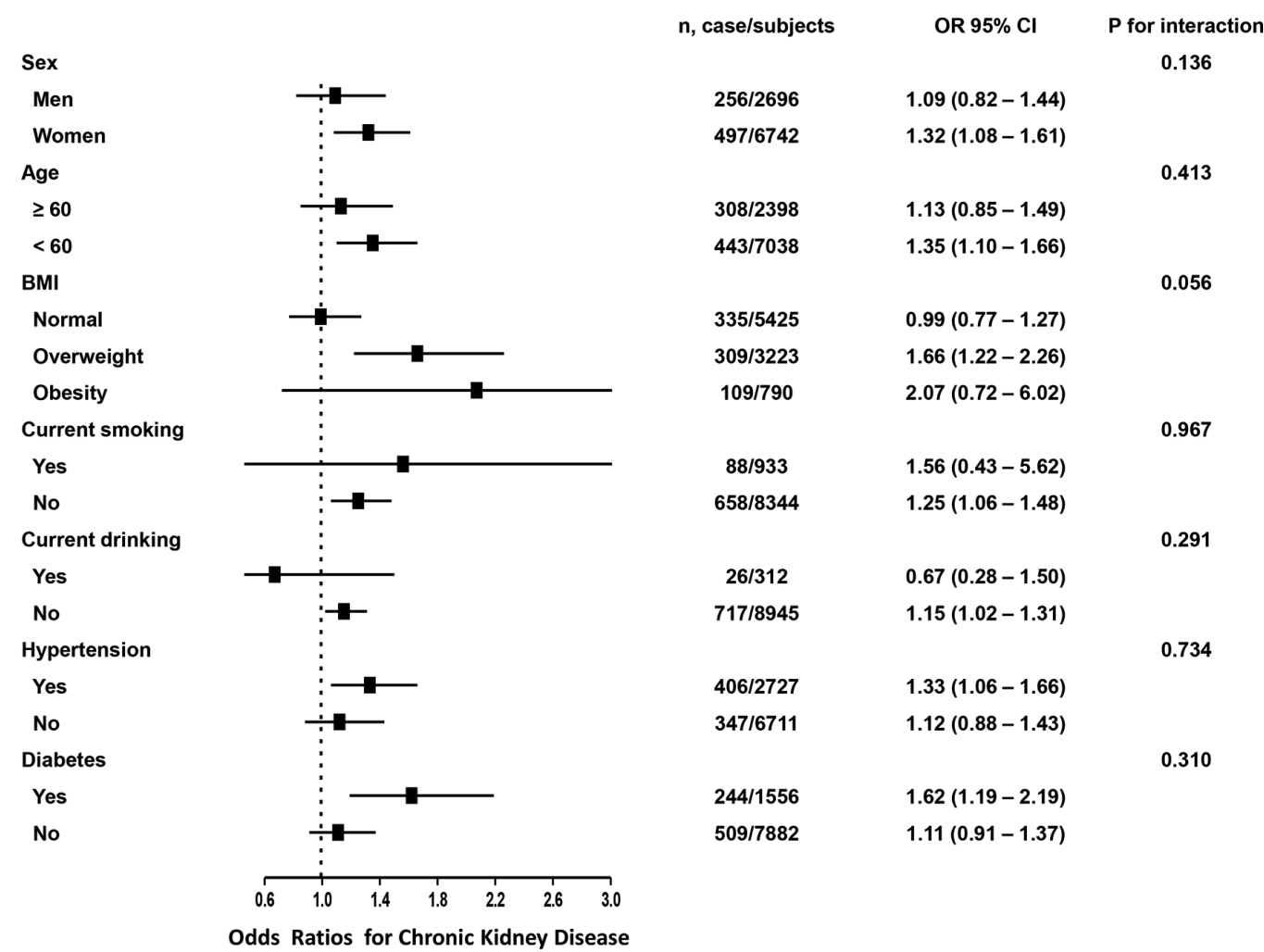

Figure 3 Risk of prevalent chronic kidney disease with each quartile increase of fatty liver index levels in different subgroups. BMI, body mass index. 
Some limitations of the study must be noted. First, owing to the observational design of the current study, we should cautiously interpret the present findings as no causal inference can be drawn. Further prospective studies are therefore needed to determine the precise relationship between FLI and risk of renal diseases. Second, by including only Chinese subjects, the results of the present study might not be representative of other ethnic groups, especially those in the developed or undeveloped countries. To some extent, however, the present study of Chinese population was still a convenience sample and selection bias is inevitable. Third, when evaluating the findings of the present study, the results should be interpreted cautiously due to possible bias from using the indirect indicator FLI to assess fatty liver disease. The calculated FLI may relate to various liver diseases with associated steatosis and not only NAFLD, despite the fact that metabolic disturbances make obesity-related steatosis likely. The internal accuracy of FLI in evaluating hepatic steatosis should also be validated using other techniques before it can be employed for these purposes. Fourth, we observed that FLI seems to play a different efficiency for kidney disease assessment in different stratifications. A significant association of FLI with increased urinary albumin excretion and CKD was only detected in subjects without current alcohol consumption. Average daily alcohol intake influences FLI, and missing such data in the present study does not permit comparisons between and within alcoholic and non-alcoholic fatty liver disease groups. To better discriminate alcoholic fatty liver disease and NAFLD, further studies need to clearly describe the precise exposure of alcohol use by collecting histories of alcohol intake in a quantitative manner. Fifth, viral hepatitis infection is one of the most serious infectious diseases worldwide, and can be associated with both liver and kidney diseases. Recent survey data showed that the hepatitis B surface antigen and anti-hepatitis $\mathrm{C}$ virus-positive rates were already $6.1 \%$ and $3.0 \%$ in China. Epidemiology of viral hepatitis infection by hepatitis B virus and hepatitis $\mathrm{C}$ virus serological testing, therefore, should also be evaluated to strengthen the findings of the present study. ${ }^{38}$ Sixth, although a spectrum of covariates was included in the adjustment, other potential mediators such as daily energy and protein intake and medicine that influence the renin-angiotensin system of the subjects should also be considered in the present study.

In conclusion, by including a large population-based cohort, the present study provides evidence that increased FLI is independently associated with prevalence of albuminuria and CKD. Findings of the present study suggested we should pay more attention to albuminuria and eGFR variation in patients with dyslipidaemia and fatty liver disease. Further prospective studies are necessary to verify our findings in external populations.
Acknowledgements We are indebted to the participants of the present study for their persistent outstanding support and to our colleagues for their valuable assistance.

Contributors Conceived and designed the experiments: LY and KS. Performed the experiments: FL, YQ, WF, CC, KS and DL. Analysed the data: KS and MR. Wrote the manuscript: KS and DL. All authors believe that the manuscript represents valid work and have reviewed and approved the final version. The work has not been published previously and is not under consideration for publication elsewhere, in part or in whole.

Funding This work was supported by grants from (1) the National Natural Science Foundation of China (81600642, 81471034, 81370910, 81300675); (2) the Natural Science Foundation of Guangdong Province, China (2015A030310433, 2017A030313831); (3) Sun Yat-sen University Medical 2016 Youth Teacher Research Funding Project (16ykpy27); (4) major project of the people's livelihood science and technology in Guangzhou (201300000102); (5) the 863 Project of Young Scientist (SS2015AA020927); (6) the Zhujiang Star of Science and Technology Foundation in Guangzhou (2014J2200046); (7) grants from the Chinese Society of Endocrinology and National Clinical Research Center for Metabolic Diseases; (8) State Key Clinical Specialty Construction Project (2011); and (9) the Science and Technology Planning Project of Guangdong Province, China (2014A020212161).

Disclaimer The funders had no role in study design, data collection and analysis, decision to publish, or preparation of the manuscript.

Competing interests None declared.

Patient consent Obtained.

Ethics approval The study protocol was approved by the Institutional Review Board of the Sun Yat-sen Memorial Hospital affiliated to Sun Yat-sen University and was in accordance with the principle of the Declaration of Helsinki II.

Provenance and peer review Not commissioned; externally peer reviewed.

Data sharing statement Main document data and additional unpublished data from the study are available by sending email to lizyhenu@163.com with proper purposes.

Open Access This is an Open Access article distributed in accordance with the Creative Commons Attribution Non Commercial (CC BY-NC 4.0) license, which permits others to distribute, remix, adapt, build upon this work non-commercially, and license their derivative works on different terms, provided the original work is properly cited and the use is non-commercial. See: http://creativecommons.org/ licenses/by-nc/4.0/

(c) Article author(s) (or their employer(s) unless otherwise stated in the text of the article) 2018. All rights reserved. No commercial use is permitted unless otherwise expressly granted.

\section{REFERENCES}

1. Matsushita K, van der Velde M, Astor BC, et al. Association of estimated glomerular filtration rate and albuminuria with all-cause and cardiovascular mortality in general population cohorts: a collaborative meta-analysis. Lancet 2010;375:2073-81.

2. Zhang L, Wang F, Wang L, et al. Prevalence of chronic kidney disease in China: a cross-sectional survey. Lancet 2012;379:815-22.

3. Matsushita K, Coresh J, Sang Y, et al. Estimated glomerular filtration rate and albuminuria for prediction of cardiovascular outcomes: a collaborative meta-analysis of individual participant data. Lancet Diabetes Endocrinol 2015;3:514-25.

4. Marcuccilli M, Chonchol M. NAFLD and Chronic Kidney Disease. Int $J$ Mol Sci 2016;17:562.

5. Musso G, Gambino R, Tabibian JH, et al. Association of nonalcoholic fatty liver disease with chronic kidney disease: a systematic review and meta-analysis. PLoS Med 2014;11:e1001680.

6. Bedogni G, Bellentani S, Miglioli L, et al. The Fatty Liver Index: a simple and accurate predictor of hepatic steatosis in the general population. BMC Gastroenterol 2006;6:33.

7. Jung $\mathrm{CH}$, Kang YM, Jang JE, et al. Fatty liver index is a risk determinant of incident type 2 diabetes in a metabolically healthy population with obesity. Obesity 2016;24:1373-9.

8. Huh JH, Ahn SV, Koh SB, et al. A prospective study of fatty liver index and incident hypertension: the KoGES-ARIRANG Study. PLOS One 2015;10:e0143560.

9. Gastaldelli A, Kozakova M, Højlund K, et al. Fatty liver is associated with insulin resistance, risk of coronary heart disease, and early 
atherosclerosis in a large European population. Hepatology 2009;49:1537-44.

10. Kozakova M, Palombo C, Eng MP, et al. Fatty liver index, gammaglutamyltransferase, and early carotid plaques. Hepatology 2012;55:1406-15.

11. Zeng J, Sun C, Sun WL, et al. Association between non-invasively diagnosed hepatic steatosis and chronic kidney disease in Chinese adults on their health check-up. J Dig Dis 2017;18:229-36.

12. Bi Y, Lu J, Wang W, et al. Cohort profile: risk evaluation of cancers in Chinese diabetic individuals: a longitudinal (REACTION) study. J Diabetes 2014;6:147-57.

13. Ning G. Reaction Study Group. Risk Evaluation of cAncers in Chinese diabeTic Individuals: a IONgitudinal (REACTION) study. $J$ Diabetes 2012;4:172-3.

14. Sun K, Li F, Qi Y, et al. Sex difference in the association between habitual daytime napping and prevalence of diabetes: a populationbased study. Endocrine 2016;52:263-70.

15. Sun K, Liu J, Ning G. Active smoking and risk of metabolic syndrome: a meta-analysis of prospective studies. PLoS One 2012;7:e47791.

16. Tomioka K, Iwamoto J, Saeki K, et al. Reliability and validity of the International Physical Activity Questionnaire (IPAQ) in elderly adults: the Fujiwara-kyo Study. J Epidemiol 2011;21:459-65.

17. Xi B, Liang $\mathrm{Y}, \mathrm{He} \mathrm{T}$, et al. Secular trends in the prevalence of general and abdominal obesity among Chinese adults, 1993-2009. Obes Rev 2012:13:287-96.

18. Ma YC, Zuo L, Chen JH, et al. Modified glomerular filtration rate estimating equation for Chinese patients with chronic kidney disease. J Am Soc Nephrol 2006;17.

19. Department of Noncommunicable Disease Surveillance. Definition, diagnosis and classification of diabetes mellitus and its complications: report of a WHO consultation. Part 1. Diagnosis and classification of diabetes mellitus. Geneva: World Health Organization, 1999. http://www.staff.ncl.ac.uk/philip.home/who_ dmg.pdf (accessed 26 Feb 2010).

20. American Diabetes Association. Standards of medical care in diabetes--2014. Diabetes Care 2014;37(Suppl 1):S14-80.

21. Huang $X, X u M, C h e n ~ Y$, et al. Validation of the fatty liver index for nonalcoholic fatty liver disease in middle-aged and elderly Chinese. Medicine 2015;94:e1682.

22. Hu L, Huang X, You C, et al. Prevalence of overweight, obesity, abdominal obesity and obesity-related risk factors in southern China. PLoS One 2017;12:e0183934.

23. Younossi ZM, Koenig AB, Abdelatif D, et al. Global epidemiology of nonalcoholic fatty liver disease-Meta-analytic assessment of prevalence, incidence, and outcomes. Hepatology 2016;64:73-84.

24. Xu C, Yu C, Ma H, et al. Prevalence and risk factors for the development of nonalcoholic fatty liver disease in a nonobese
Chinese population: the Zhejiang Zhenhai Study. Am J Gastroenterol 2013;108:1299-304.

25. Middleton MS, Heba ER, Hooker CA, et al. Agreement between magnetic resonance imaging proton density fat fraction measurements and pathologist-assigned steatosis grades of liver biopsies from adults with nonalcoholic steatohepatitis. Gastroenterology 2017;153:753-61.

26. Hernaez R, Lazo M, Bonekamp S, et al. Diagnostic accuracy and reliability of ultrasonography for the detection of fatty liver: a metaanalysis. Hepatology 2011;54:1082-90.

27. Sun K, Lu J, Jiang Y, et al. Low serum potassium level is associated with nonalcoholic fatty liver disease and its related metabolic disorders. Clin Endocrinol 2014;80:348-55.

28. Karanjia RN, Crossey MM, Cox IJ, et al. Hepatic steatosis and fibrosis: Non-invasive assessment. World J Gastroenterol 2016;22:9880-97.

29. Lee YH, Jung KS, Kim SU, et al. Sarcopaenia is associated with NAFLD independently of obesity and insulin resistance: Nationwide surveys (KNHANES 2008-2011). J Hepatol 2015;63:486-93.

30. Yang BL, Wu WC, Fang KC, et al. External validation of fatty liver index for identifying ultrasonographic fatty liver in a large-scale cross-sectional study in Taiwan. PLoS One 2015;10:e0120443.

31. Sun K, Lin D, Li F, et al. Discordant associations of lipid parameters with albuminuria and chronic kidney disease: a population-based study. Lipids Health Dis 2015;14:152.

32. Gaggini M, Morelli M, Buzzigoli E, et al. Non-alcoholic fatty liver disease (NAFLD) and its connection with insulin resistance, dyslipidemia, atherosclerosis and coronary heart disease. Nutrients 2013:5:1544-60.

33. Meex RCR, Watt MJ. Hepatokines: linking nonalcoholic fatty liver disease and insulin resistance. Nat Rev Endocrinol 2017;13:509-20.

34. Giorda C, Forlani G, Manti R, et al. Occurrence over time and regression of nonalcoholic fatty liver disease in type 2 diabetes. Diabetes Metab Res Rev 2017;33:e2878.

35. Parker R, Kim SJ, Gao B. Alcohol, adipose tissue and liver disease: mechanistic links and clinical considerations. Nat Rev Gastroenterol Hepatol 2018;15:50-9.

36. Schrieks IC, Heil AL, Hendriks HF, et al. The effect of alcohol consumption on insulin sensitivity and glycemic status: a systematic review and meta-analysis of intervention studies. Diabetes Care 2015;38:723-32.

37. Koning SH, Gansevoort RT, Mukamal KJ, et al. Alcohol consumption is inversely associated with the risk of developing chronic kidney disease. Kidney Int 2015;87:1009-16.

38. Zhang Q, Qi W, Wang X, et al. Epidemiology of Hepatitis B and Hepatitis $C$ infections and benefits of programs for hepatitis prevention in northeastern China: a cross-sectional study. Clin Infect Dis 2016;62:305-12. 